\title{
Carnitine and congeners as regulators of tumour necrosis factor
}

In the past few years the steady increase in septic shock has been fuelled by the augmented use of invasive medical technology, immunosuppressive drug therapy, and increased longevity of patients with secondary immunodeficiencies.

The septic shock syndrome probably represents the effects of many mediators on vasculature, myocardium and other systems. Endotoxin has been postulated to mediate the early stages of mediator cascade during septic shock, and inhibiting its effect has become an underlying principle of certain therapies. The presently available anti-endotoxin monoclonal antibodies seem effective only in patients with Gram-negative bacterial infections. However, there is usually no time to distinguish this sub-group of individuals from others with different infections before the start of therapy and one of the central problems with antibody-based therapies - the lack of understanding of how they work-is still unsolved.

Tumour necrosis factor might be a direct or indirect mediator of many of the cardiovascular responses associated with sepsis. Hypotension, hypothermia, hypoglycaemia, acidosis, focal hepatic necrosis, and acute interstitial pneumonitis with neutrophil trapping have been observed following experimental injections of high doses of TNF. As a mediator of inflammation, TNF triggers the release of a large body of cytokines, interleukin 1 included, and of arachidonic acid metabolites. Experimental data have suggested that TNF could be an appropriate target for the treatment of septic shock, but work in animals up to now has shown that anti-TNF antibodies are not very effective in focal sepsis.

Recently, many investigators independently from each other reached the conclusion that carnitine and congeners can behave as modulators of TNF production. The results of their studies were the subject of a symposium held in Pomezia, Rome, on 28 January, 1993 and are published in Mediators of Inflammation.

The information presented in this volume must be considered as an effort to stimulate scientists' creative thought and clinicians' awareness of the therapeutic potential of carnitine and carnitine congeners for the treatment of septic shock.

\section{Guest Editors}

Claudio De Simone Edoardo Arrigoni Martelli Piero Foresta 


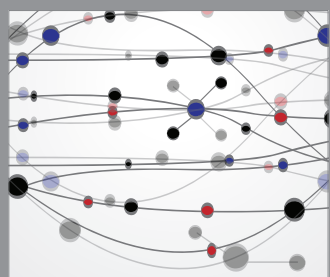

The Scientific World Journal
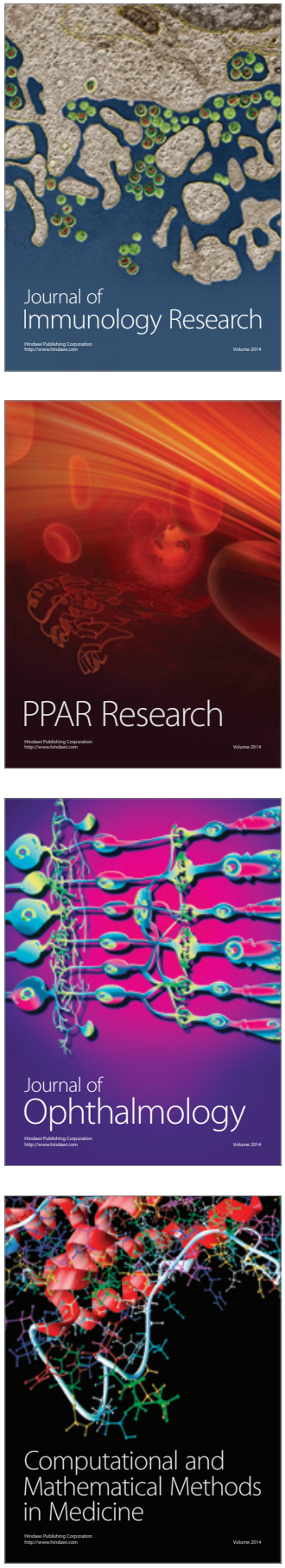

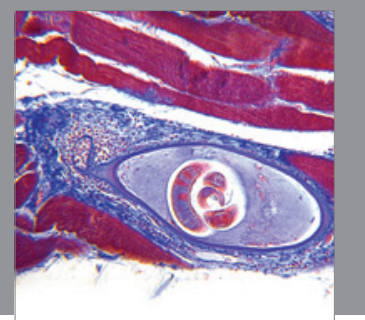

Gastroenterology

Research and Practice
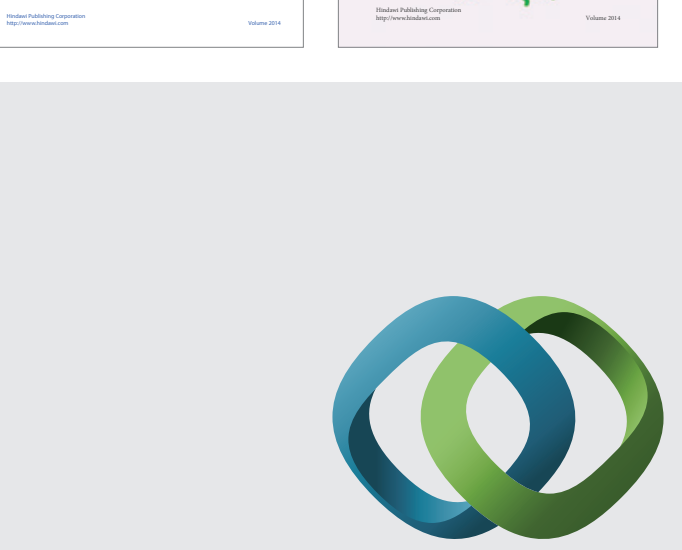

\section{Hindawi}

Submit your manuscripts at

http://www.hindawi.com
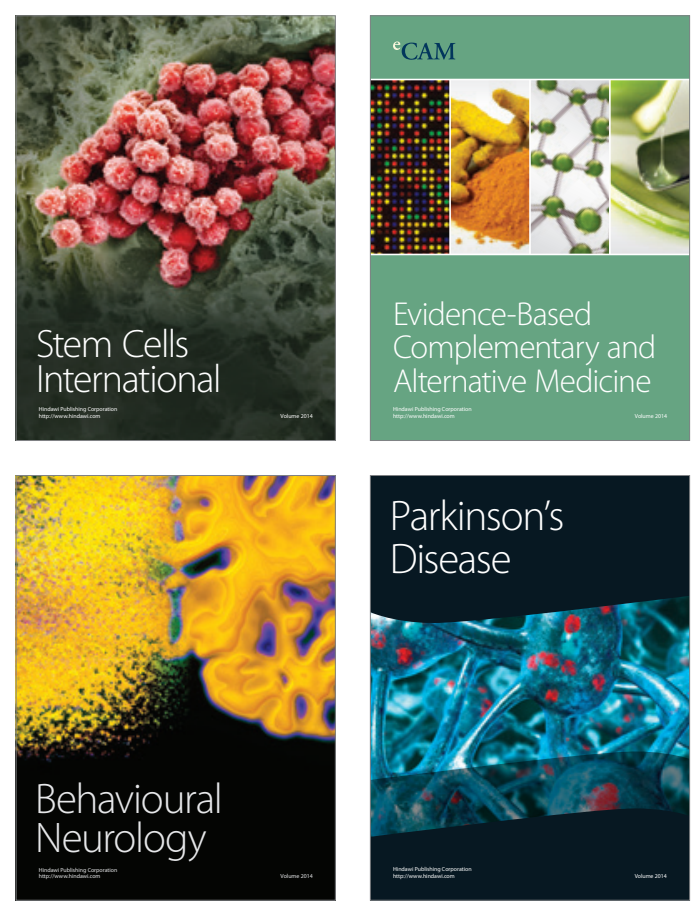

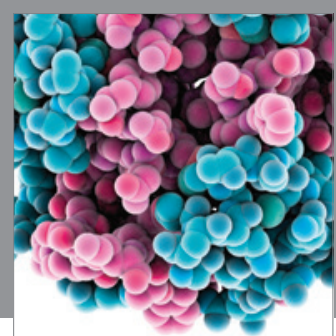

Journal of
Diabetes Research

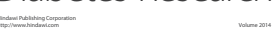

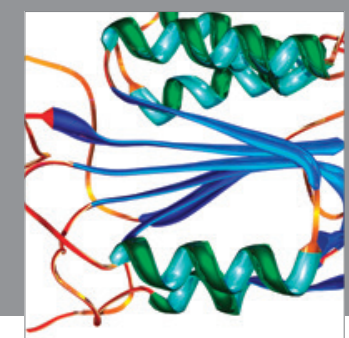

Disease Markers
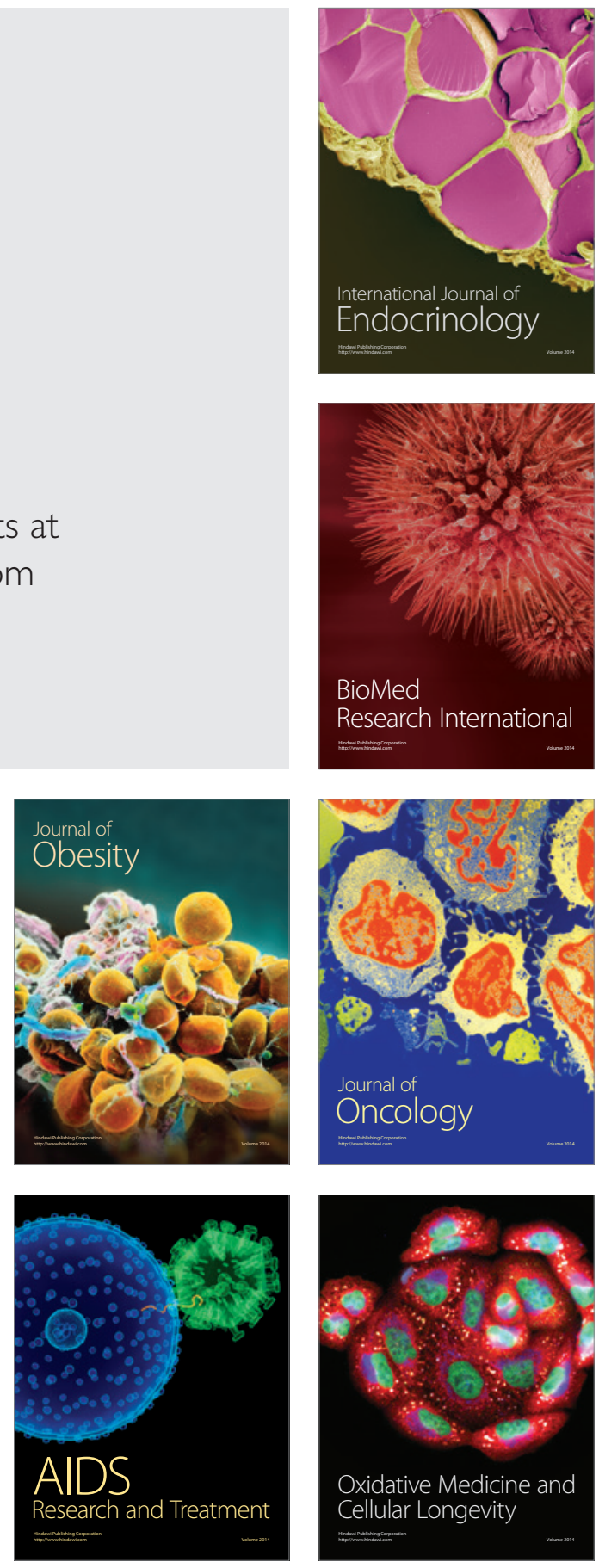